\title{
DISTRIBUIÇÃO GEOGRÁFICA DA LEISHMANIOSE TEGUMENTAR AMERICANA NO ESTADO DO ESPÍRITO SANTO - BRASIL
}

\author{
Paulo Augusto Sessa, Gelcilio Coutinho Barros, Enilse Antunes de Mattos, \\ Valquiria Rocha Daher Carias, José Tasso Aires de Alencar, \\ Delio Delmaestro, Carlos Cley Coelho e Aloisio Falqueto.
}

\begin{abstract}
Com base nos registros de 730 pacientes portadores de Leishmaniose Tegumentar Americana (LTA) referentes ao periodo de janeiro de 1972 a dezembro de 1982, os autores realizaram um estudo da distribuiçáo geográfica dessa parasitose no Estado do Espirito Santo. Os pacientes procediam de 36 dos 53 municípios que compóem o Estado, destacando-se Viana e Cariacica como os de maior prevalência, somando juntos 442 casos $(60,54 \%)$, com 259 destes residindo numas poucas localidades, contínuas entre si, formando uma área endêmica, com transmissão ocorrendo provavelmente no peri e intra-domicilio. Esta área de alta endemicidade, pertencente principalmente ao vale do rio Formate, estende-se também ao município de Domingos Martins através da localidade de Biriricas. Nos demais municipios a LTA caracterizouse como uma doença profissional de ocorrência antiga no Estado.
\end{abstract}

Palavras chaves: Leishmaniose tegumentar americana. Distribuição geográfica.

Após Cunha ${ }^{4}$, Carini $^{3}$, Terra ${ }^{15}$ e Silva ${ }^{14}$ terem demonstrado a existência da Leishmaniose Tegumentar Americana no Estado do Espirito Santo, longo periodo se passou até Barros e cols ${ }^{2}$ descreverem um foco da doença abrangendo parte dos municípios de Viana e Cariacica. Para estudar esse foco a Disciplina de Parasitologia da Universidade Federal do Espírito Santo criou um ambulatório que atendia também a todos os casos encaminhados à Superintendência de Campanhas do Ministério da Saúde para tratamento, além dos pacientes trazidos diretamente por pessoas que conheciam o serviço, algumas das quais anteriormente tratadas.

Durante todo o tempo que o ambulatório funcionou foram atendidos pacientes oriundos de diversos municipios do Estado, o que permitiu o presente levantamento.

\section{MATERIAL E MÉTODOS}

No periodo de maio de 1978 a dezembro de 1982 a Disciplina de Parasitologia da Universidade Federal do Espírito Santo relacionou 311 pacientes que contrairam a Leishmaniose Tegumentar Americana (LTA) nos próprios municipios de residência no Estado. O diagnóstico foi estabelecido isoladamente ou em associação pelo aspecto clínico das lesões,

Trabalho da Disciplina de Parasitologia do Departamento de Patologia do Centro Biomédico da Universidade Federal do Espírito Santo e da Superintendência de Campanhas do Ministério da SaúdeRegional do Espirito Santo.

Departamento de Patologia do Centro Biomédico da Universidade Federal do Espirito Santo. Av. Marechal Campos 1468, Maruipe - 29.000 Vitória, ES. Brasil.

Recebido para publicação em $27 / 8 / 1984$ pela Intradermorreação de Montenegro (IRM) e pelo exame de impressões em lâminas e cortes histológicos preparados a partir de biópsias. Na IRM utilizou-se o antigeno fornecido pelo Departamento de Parasitologia do Instituto de Ciências Biológicas da Universidade Federal de Minas Gerais, padronizado segundo a técnica de Melo e cols ${ }^{11}$.

Adicionalmente foram também considerados os 419 casos registrados pela Superintendência de Campanhas do Ministério da Saúde - Regional do Espírito Santo (SUCAM-ES) de janeiro de 1972 a abril de 1978. Nesses registros não há indicação dos métodos diagnósticos nem da forma clínica.

\section{RESULTADOS}

A Tabela 1 mostra a distribuição anual dos 311 pacientes atendidos pela Disciplina de Parasitologia da UFES. Observa-se que os municipios com maior prevalência foram Viana e Cariacica, seguidos por Guarapari, Domingos Martins, Colatina e Linhares. A situação geográfica de todos os municípios do Estado está na Figura 1. Analisando-se a procedência dos pacientes nesses 6 municipios verificou-se que em Viana, Cariacica e Domingos Martins a maioria deles residia numas poucas localidades, contínuas entre si, como pode ser visto na Tabela 2 e Figura 2. Na Tabela 3 tem-se a distribuição dos casos ocorridos naqueles 6 municípios e nos demais em função da idade, sexo e formas clinicas.

Dentre os 419 casos registrados pela SUCAMES 255 (60,85\%) ocorreram nos municípios de Viana e Cariacica. Dos 87 de Viana, 37 procediam da localidade de Perobas e 5 de Formate; dos 168 de Cariacica 37 eram de Piranema, 21 de Boa Vista, 15 de Roda D'água e 9 de Duas Bocas. Nesses dois municipios 54 apresentavam idade de $0-10$ anos, 65 de $11-20$ e 136 acima de 20 anos. Ao sexo masculino pertenciam 144 e ao feminino 111 . 
Sessa PA, Barros GC, Mattos EA, Carias VRD, Alencar JTA, Delmaestro D, Coelho CC, Falqueto A. Distribuição geográfica da leishmaniose tegumentar americana no Estado do Espírito Santo-Brasil. Revista da Sociedade Brasileira de Medicina Tropical 18: 237-241, Out-Dez, 1985

Tabela 1 -Distribuição dos 311 pacientes portadores de Leishmaniose Tegumentar Americana em funcão do municipio de procedência e do ano em que foram diagnosticados.

\begin{tabular}{|c|c|c|c|c|c|c|c|}
\hline \multirow[b]{2}{*}{ Munictpios } & \multicolumn{5}{|c|}{ Anos } & \multirow[b]{2}{*}{ Tolal } & \multirow[b]{2}{*}{$\%$} \\
\hline & 1978 & 1979 & 1980 & 1981 & 1982 & & \\
\hline Afonso Claudio & . & 1 & 2 & - & - & 3 & 0.96 \\
\hline Alegre & & - & - & 1 & - & 1 & 0.32 \\
\hline Baixo Guandu & - & 1 & 1 & - & - & 2 & 0.64 \\
\hline Barra de Sào Franciseo & - & 1 & - & 1 & 1 & 3 & 0.96 \\
\hline Cachociro de Itapemirim & - & & 1 & - & - & 1 & 0.32 \\
\hline Cariacica & 17 & 23 & 29 & 12 & 5 & 86 & 27.65 \\
\hline Castelo & - & - & - & 1 & - & 1 & 0.32 \\
\hline Colatina & - & 1 & 9 & 1 & 1 & 12 & 3.85 \\
\hline Conceição da Barra & - & 1 & - & & - & 1 & 0.32 \\
\hline Domingos Marins & 3 & 1 & 9 & 3 & & 16 & 5.14 \\
\hline Ecoporanga & & 2 & - & - & - & 2 & 0.64 \\
\hline Fundảo & - & & 2 & 3 & 1 & 6 & 1.92 \\
\hline Guaçuı & - & - & 1 & - & - & l & 0.32 \\
\hline Guarapari & .. & 10 & 2 & 3 & 2 & 17 & 5.46 \\
\hline Ibiracu & - & 1 & - & l & 4 & 6 & 1.92 \\
\hline Itaguaçu & & 2 & 1 & & - & 3 & 0.96 \\
\hline Itarana & - & 4 & - & & 1 & 5 & 1.60 \\
\hline luna & & 2 & 3 & 1 & - & 6 & 1.92 \\
\hline Linhares & 2 & 4 & - & 2 & 2 & 10 & 3.21 \\
\hline Nosa Venecia & 1 & - & - & 1 & & 2 & 0.64 \\
\hline Pancas & 1 & - & - & - & - & 1 & 0.32 \\
\hline Pinheiro & & 1 & - & & 1 & 2 & 0.64 \\
\hline Santa Leo & 1 & 3 & 2 & 1 & - & 7 & 2.25 \\
\hline Sāo Gabriel da Palha & 1 & - & & - & - & 1 & 0.32 \\
\hline Sào Mateus & - & 2 & l & 2 & -- & 5 & 1.60 \\
\hline Serra & - & - & 1 & - & 2 & 3 & 0.96 \\
\hline Viana & 39 & 16 & 11 & 1.3 & 22 & 101 & 32.47 \\
\hline Vila Velha & - & 2 & - & 2 & 1 & 5 & 1.60 \\
\hline Vitoria & - & 1 & - & - & 1 & 2 & 0.64 \\
\hline$\overline{\text { Total }}$ & 65 & 79 & 75 & 48 & 44 & 311 & 100.00 \\
\hline
\end{tabular}

\section{DISCUSSĀO}

A análise conjunta dos registros feitos pela Disciplina de Parasitologia da UFES e pela SUCAMES, no período de janeiro de 1972 a dezembro de 1982 , demonstrou que os 730 pacientes procediam de 36 dos 53 municípios que compõem o Estado?, destacando-se Viana e Cariacica como os de maior prevalència, somando juntos 442 casos $(60,54 \%)$, com 259 destes $(58,59 \%)$ residindo em poucas localidades próximas umas das outras, formando uma
Tabela 2-Distribuição dos casos de Leishmaniose Tegumentar Americana ocorridos nos municipios de Viana, Cariacica e Domingos Martins, em função das localidades mais atingidas e do ano em que foi feito o diagnóstico.

\begin{tabular}{|c|c|c|c|c|c|c|c|}
\hline \multirow[b]{2}{*}{ Mumicipios } & \multirow[b]{2}{*}{ Localidades } & \multicolumn{5}{|c|}{ Anos } & \multirow[b]{2}{*}{ Total } \\
\hline & & 1978 & 1979 & 1980 & 1981 & 1982 & \\
\hline \multirow[t]{7}{*}{$\overline{\text { Viana }}$} & Peroba & 18 & 5 & - & 2 & 1 & $\overline{26}$ \\
\hline & Formate * & 10 & 5 & 1 & 2 & 5 & 23 \\
\hline & Coacas & 6 & & 1 & - & 5 & 12 \\
\hline & Biriricas ** & $\ldots$ & & 7 & 4 & & 11 \\
\hline & Bairo Universat & _- & .. & 1 & 2 & 5 & 8 \\
\hline & Outras & 5 & 6 & 1 & 3 & 6 & 21 \\
\hline & Total & 39 & 16 & 11 & 13 & 22 & 101 \\
\hline \multirow[t]{9}{*}{ Cariacica } & Roda $D^{\circ}$ Agua & 11 & 1 & 2 & 4 & I & 19 \\
\hline & Duas Bocas & - & 6 & 2 & - & - & 8 \\
\hline & Rocinha & & & & & & \\
\hline & (Bairro Nowo Brasil) & 1 & 3 & 3 & - & - & 7 \\
\hline & Boa Vista & 2 & 3 & - & 1 & 1 & 7 \\
\hline & Piranema & - & & 7 & - & & 7 \\
\hline & Formate * & - & 5 & 1 & 1 & & \\
\hline & Outras & 3 & 5 & 14 & 6 & 3 & 31 \\
\hline & Total & 17 & 23 & 29 & 12 & 5 & 86 \\
\hline \multirow{4}{*}{$\begin{array}{l}\text { Domingos } \\
\text { Martins }\end{array}$} & & & & & & & \\
\hline & Biriricas ** & - & - & .- & g & 2 & 11 \\
\hline & Outras & - & 3 & 1 & & I & \\
\hline & Total & - & 3 & 1 & 9 & 3 & 16 \\
\hline
\end{tabular}

* Localidade situada nos municipios de Cariacica e Viana

** Localidade situada nos municipios de Viana e I Domingos Martins.

área endêmica (Tabelas 1 e 2 e Figura 1 e 2). Embora não se tenha relato anterior a 1972 é possivel que nesses dois municipios o numero de pessoas acometidas pela LTA tenha começado a aumentar no inicio da década de 1970 como sugerem a Figura 3 e os poucos casos com lesões mucosas diagnosticados no periodo de maio de 1978 a dezembro de 1982 (Tabela 3). $\mathrm{O}$ fato de ambos os municípios pertencerem à microrregião homogênea de Vitória, situarem-se próximos ao litoral entre $20^{\circ} 13^{\prime}$ e $20^{\circ} 31^{\prime}$ S e entre $40^{\circ} 20^{\prime}$ e $40^{\circ} 37 \mathrm{~W}$, e serem descontínuos com áreas endèmicas de importância indica que surgiram internamente condições favoráveis à trasmissão que provavelmente seriam:

Tabela 3 - Distribuição dos 311 pacientes portadores de Leishmaniose Tegumentar Americana diagnosticados no periodo de maio de 1978 a dezembro de 1982 em função da idade, sexo e formas clínicas, com destaque para os municipios com maior número de casos.

\section{Faixa Etária}

Sexo

Formas Clinicas

Municipios $\quad 0-5 \quad 6-1011-1516-20>20$ n! Masculino Feminino Cutâneo Mucosa Cutâneo-

\begin{tabular}{lrrrrrrrrrrr} 
& & & 10 & & & & & Mucosa \\
\hline Viana & 12 & 16 & 21 & 3 & 49 & 101 & 58 & 43 & 95 & 5 & 1 \\
Cariacica & 14 & 12 & 10 & 4 & 46 & 86 & 53 & 33 & 74 & 6 & 6 \\
Guarapari & 4 & 3 & 3 & 1 & 6 & 17 & 10 & 7 & 15 & 2 & - \\
Domingos Martins & 2 & 1 & 1 & 2 & 10 & 16 & 9 & 7 & 15 & 1 & - \\
Colatina & - & 1 & 5 & 1 & 5 & 12 & 7 & 5 & 11 & 1 & - \\
Linhares & - & & - & 1 & 9 & 10 & 8 & 2 & 5 & 5 & - \\
Outras & 2 & 4 & 1 & 10 & 52 & 69 & 50 & 19 & 49 & 18 & 2 \\
\hline Total & 34 & 37 & 41 & 22 & 177 & 311 & 195 & 116 & 264 & 38 & 9 \\
\hline
\end{tabular}


Sessa PA, Barros GC, Mattos EA, Carias VRD, Alencar JTA, Delmaestro D, Coelho CC, Falqueto A. Distribuição geográfica da leishmaniose tegumentar americana no Estado do Espirito Santo-Brasil. Revista da Sociedade Brasileira de Medicina Tropical 18: 237-241, Out-Dez, 1985

FigURA 1 - MAPA DO ESTADO DO ESPIRITO SANTO - DIVISẢo POLITICA

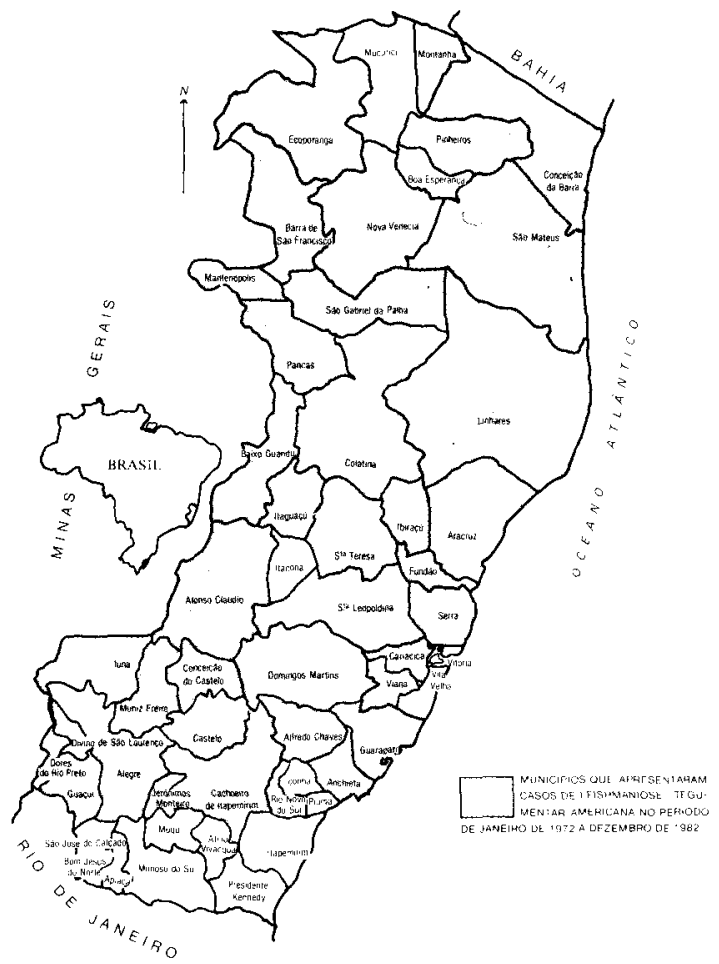

Fonte: Disciplina de Parasitologia da Universidade Federaı do Espírito Santo e Superintendència de Campanhas do Ministério da Saide-Regional do Espirito Santo.

a) A existência da reserva florestal de Duas Bocas que funcionou como centro de difusão do parasito.

b) A substituição dos cafezais por culturas de banana no final da década de 1960 que permitiu, após alguns anos, a instalação de uma fauna de mamiferos e flebotomíneos própria ao novo ambiente e que trouxe consigo o parasita, permitindo a transmissão ao homem. A ocorrência de LTA em áreas de bananais já foi registrada por Sabroza ${ }^{13}$, Menezes ${ }^{12}$ e Araújo Filho'.

c) A manutenção de um enzootia peios cães domésticos que Falqueto ${ }^{5}$ demonstrou existir no foco descrito por Barros e cols ${ }^{2}$, que abrange parte desses dois municipios.

d) $\mathrm{O}$ estabelecimento de uma transmissão no peri e intradomicílio, visto o acometimento elevado de individuo do sexo feminino e de baixa idade como já referido por Barros e cols ${ }^{2}$ em virtude da enzootia canina (Falqueto ${ }^{5}$ ) e da localização das casas em meio aos bananais. Barros e cols ${ }^{2}$. Fala a favor também dessa transmissão o fato de Mattos ${ }^{9}$ ter verificado que na localidade de Perobas, municipio de Viana, os flebotomíneos são mais abundantes nesses dois ambientes, sendo o
Figura 2-Mapa dos municipios de Cariacica e Viana mostrando as localidades que apresentaram maior número de casos de Leishmaniose Tegumentar Americana no periodo de janeiro de 1972 a dezembro de 1982.

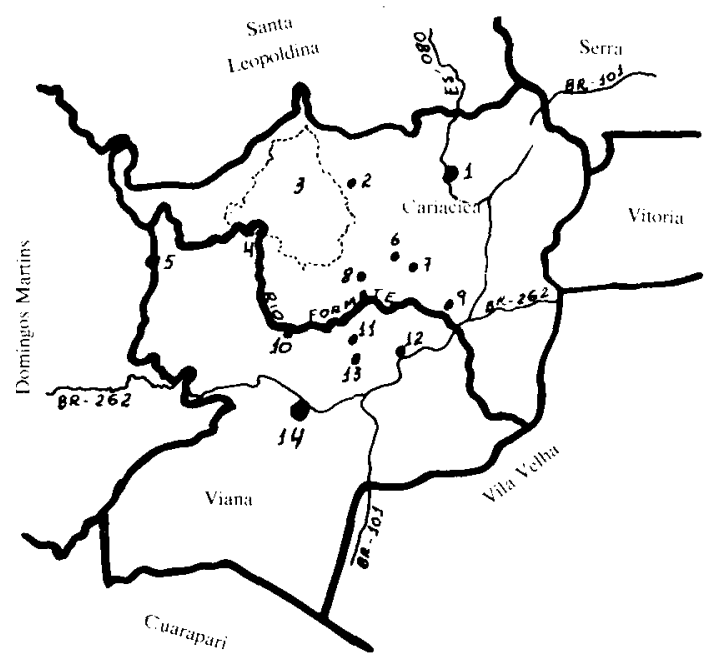

1 - Sede do município de Cariacica

2 - Duas Bocas

3 - Reserva florestal de Duas Bocas

4 - Nascentes do Rio Formate

5 - Biriricas

6 - Boa Vista

7 - Novo Brasil

8 - Roda D'Água

9 - Piranema

10 - Formate

11 - Coaca

12 - Bairro Universal

13 - Peroba

14 - Sede do município de Viana

Fonte: Disciplina de Parasitologia da Universidade Federal do Espirito Santo e Superintendência de Campanhas do Ministerio da Saude-Regional do Espirito Santo.

Lutzomya intermedia a espécie mais freqüente. Este flebotomíneo, segundo Forattini e cols 6 e Gomes $^{8}$ apud Tolezano e cols ${ }^{16}$, no Estado de São Paulo exibe um comportamento antropofílico e domiciliar.

Esta área de alta endemicidade, pertencente principalmente ao vale do rio Formate, estendeu-se também ao município de Domingos Martins através da localidade de Biriricas, na qual ocorreram 11 $(68,75 \%)$ dos 16 casos registrados pela Disciplina de Parasitologia da UFES (Tabela 2 e Figura 2).

Embora no município de Guarapari os 17 casos assinalados nos anos de 1979 a 1982 residissem em áreas de plantio de banana, e $10(58,82 \%)$ tivessem 
Sessa PA, Barros GC, Mattos EA, Carias VRD, Alencar JTA, Delmaestro D, Coelho CC, Falqueto A. Distribuição geográfica da leishmaniose tegumentar americana no Estado do Espírito Santo-Brasil. Revista da Sociedade Brasileira de Medicina Tropical 18: 237-241, Out-Dez, 1985

Figura 3 - Distribuição por ano dos casos de Leishmaniose Tegumentar Americana ocorridos nos municipios de Cariacica e Viana no periodo de janeiro de 1972 a dezembro de 1982.

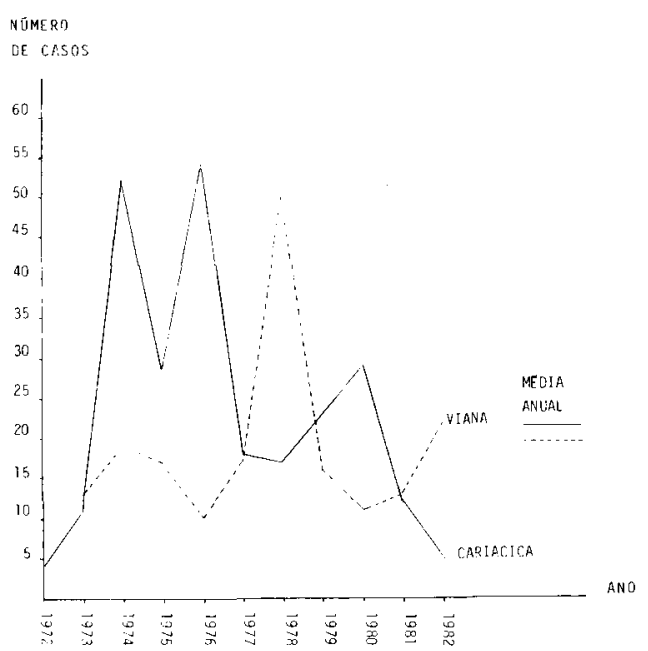

Fonte: Disciplina de Parasitologia da Universidade Federal do Espírito Santo e Superintendência de Campanhas do Ministério da Saúde-Regional do Espirito Santo.

até 15 anos, as localidades não eram contínuas entre si nem com as de Viana e Domingos Martins.

Em cada um dos demais municípios de ocorrência a LTA acometeu poucas pessoas e de maneira esporádica ao longo dos anos, sendo a maioria adultos do sexo masculino e com grande número de formas mucosas, caracterizando uma doença profissional e antiga no Estado. Neste contexto incluem-se também os municipios do Vale do Rio Doce (Linhares, Colatina, Baixo Guandu, Itarana, Itaguaçu, Pancas, Afonso Clláudio e São Gabriel da Palha), apesar de no Estado de Minas Gerais existir área endêmica de importância na microrregião da Mata de Caratinga, situada também no vale, descrita por Mayrink e cols 10 .

O não registro da LTA em vários municípios do sul do Espírito Santo (Figura 1 ) pode ser explicado ou pela não ocorrência real ou por ser mais fácil para a população procurar tratamento no Estado do Rio de Janeiro.

\section{SUMMARY}

Analysis of the case records of 729 patients with cutaneous leishmaniasis collected from January 1972 to December 1982 permitted the authors to study the geographical distribution of this infection in the State of Espirito Santo, Brazil. Cases were found in
36 of the 53 counties of this State, the counties of Viana and Cariacica being the ones with greatest incidence of the disease. The cases observed in these two counties totalled $441(60,47 \%)$ with 258 patients living in a few contiguous small villages in the endemic region. The transmission probably occurred inside and outside the house. From this highly endemic area in the valley of the Formate river disease has already spreaded up to Domingos Martins county through the village of Biriricas.

In the other regions cutaneous leishmanlasis was characterised as a longstanding disease in the State.

Key words: American cutaneous leishmaniasis. Geographic distribution.

\section{REFERÊNCIAS BIBLIOGRÁFICAS}

1. Araújo Filho NA. Epidemiologia da leishmaniose tegumentar americana na Ilha Grande, Rio de Janeiro. Estudos sobre a infecção humana, reservatórios e transmissores. Tese de Mestrado. Universidade Federal do Rio de Janeiro, Rio de Janeiro, 1978.

2. Barros GC, Sessa PA, Mattos EA, Carias VRD, Mayrink W, Alencar JTA, Falqueto A, Jesus AC. Foco de leishmaniose tegumentar americana nos municípios de Viana e Cariacica, Estado do Espirito Santo, Brasil. Revista de Saúde Pública 19: 146-153, 1985.

3. Carini A. Um novo caso de leishmaniose das mucosas. Archivos da Sociedade de Medicina e Cirurgia de São Paulo 14: 364-66, 1912.

4. Cunha C. Comunicação à sessão de 28 de julho ủa Sociedade Brasileira de Dermatologia 1: 42, 1912.

5. Falqueto A. Leishmaniose tegumentar em Viana, Estado do Espírito Santo. Investigação sobre infecçāo natural em animais e sua relação com a ocorrência da doença humana. Tese de Mestrado da Universidade Federal do Rio de Janeiro, Rio de Janeiro, 1984.

6. Forattini OP, Rabello EX, Serra OP, Contrin MD, Galati EAB e Barata JMS. Observações sobre a transmissão da Leishmaniose tegumentar no Estado de São Paulo, Brasil. Revista de Saúde Pública 10: 31-43, 1976.

7. Fundação Instituto Brasileiro de Geografia e Estatística. Sinopse preliminar do censo demográfico. In: IX Recenseamento Geral do Brasil 16, 1980.

8. Gomes AO. Observaçòes ecológicas sobre o Psychodopygus intermedius no vale do Ribeira, Estado de São Paulo, Brasil. Tese de Mestrado da Universidade de São Paulo, São Paulo, 1979.

9. Mattos EA. Bionomia dos flebotomineos de Perobas, municipio de Viana (ES), área endêmica de Leishmaniose Tegumentar Americana. Tese de Mestrado da Universidade Federal de Minas Gerais, 1981.

10. Mayrink M, Willians P, Coelho MV, Dias M, Martins AV, Magalhães PA, Costa CA, Falcão AR, Melo MN, Falcão AL. Epidemiology of dermal leishmaniasis in the Rio Doce Valley, State of Minas Gerais, Brazil. Annals of Tropical Medicine and Parasitology 73: 123$137,1979$.

11. Melo MN, Mayrink M, Costa CA, Magalhães P, Dias M., Williams P, Araújo FG, Coelho MV, Batista SM. Padronizacão do antigeno de Montenegro. Revista do Instituto de Medicina Tropical de São Paulo 19: 161164, 1977. 
Sessa PA, Barros GC, Mattos EA, Carias VRD, Alencar JTA, Delmaestro D, Coelho CC, Falqueto A. Distribuição geográfica da leishmaniose tegumentar americana no Estado do Espirito Santo-Brasil. Revista da Sociedade Brasileira de Medicina Tropical 18: 237-241, Out-Dez, 1985

12. Menezes JA. Leishmaniose tegumentar no Estado do Rio de Janeiro. Inquéritos por intradermorreação. Tese de Mestrado da Universidade Federal do Rio de Janeiro, Rio de Janeiro, 1976.

13. Sabroza PA. O domicílio como fator de risco na leishmaniose tegumentar americana. Estudo epidemiológico em Jacarepaguá, municipio do Rio de Janeiro. Tese de Mestrado da Escola de Saúde Pública, 1983.
14. Silva OD. Sobre a leishmaniose tegumentar e seu tratamento. Memórias do Instituto Oswaldo Cruz 7: 213-48, 1915 .

15. Terra F. Leishmaniose tegumentaire au Brésil. Boletim da Sociedade Brasileira de Dermatologia 2: 58-67, 1913.

16. Tolezano JE, Macoris SA, Diniz JMP. Modificaçōes na Epidemiologia da leishmaniose tegumentar no Vale da Ribeira, Estado de São Paulo, Brasil. Revista do Instituto Adolfo Lutz 40: 49-54, 1980. 